\title{
Antineoplastic behavior of polydopamine nanoparticles prepared in different water/alcohol media
}

\author{
Celia Nieto $^{a}$, Gema Marcelo ${ }^{\mathrm{b}}$, Milena Vega ${ }^{\mathrm{a}, *}$, Eva M. Martín del Valle ${ }^{\mathrm{a}, *}$ \\ ${ }^{a}$ Departamento de Ingeniería Química y Textil, Facultad de Ciencias Químicas, Universidad de Salamanca, 37008, Salamanca, Spain \\ ${ }^{\mathrm{b}}$ Departamento de Química Analítica, Química Física e Ingeniería Química, Facultad de Farmacia, Universidad de Alcalá, 28801, Alcalá de Henares (Madrid), Spain
}

\section{A R T I C L E I N F O}

\section{Keywords:}

Polydopamine nanoparticles

Alcohols

Size

Iron adsorption capacity

Cytotoxicity

\begin{abstract}
A B S T R A C T
Polydopamine nanoparticles (PD NPs) have been synthesized in the present work through the oxidative polymerization of dopamine in aqueous media containing five different types of alcohol in a constant solvent volume ratio. We have shown that the type of alcohol, along with the ammonium hydroxide concentration used in the synthesis process, conditions particle size. Additionally, it has been found that the type of alcohol employed influences the well-known capacity of polydopamine nanoparticles to adsorb iron. As a consequence, since a ferroptosis-like mechanism may account for the cytotoxicity of these nanoparticles, the type of alcohol could also have a determining role in their antineoplastic activity. Here, the existence of a correlation between the ability of polydopamine nanoparticles to load $\mathrm{Fe}^{3+}$ and their toxic effect on breast cancer cells has been proven. For instance, nanoparticles synthesized using 2-propanol adsorbed more $\mathrm{Fe}^{3+}$ and had the greatest capacity to reduce breast tumor cell viability. Moreover, none of the nanoparticle synthesized with the different alcohols significantly decreased normal cell survival. Cancer cells present greater iron-dependence than healthy cells and this fact may explain why polydopamine nanoparticles toxicity, in which Fenton chemistry could be implicated, seems tumor-specific.
\end{abstract}

\section{Introduction}

Polydopamine, a synthetic melanin that made its way into materials Science as a coating agent [1], has begun to gain importance for cancer diagnosis and therapy [2]. Like natural melanins, which play an essential role in our organism by regulating the concentration of free metal cations $[3,4]$, polydopamine has also shown to have a great affinity for such ions [5]. For this reason, nanoparticles synthesized with this polymer are being developed to create novel contrast agents for imaging techniques and new photothermal therapy systems $[6,7]$.

Likewise, it has been observed that polydopamine nanoparticles (PD NPs) have intrinsic antineoplastic activity, and this property could be also related to their affinity for a metal cation: $\mathrm{Fe}^{3+}$. When internalized, PD NPs end up in lysosomes, the organelles that precisely store free cations in the cell, and it is though that, given their high $\mathrm{Fe}^{3+}$-loading capacity compared to that of other cations, they may be involved in a ferroptosis-like mechanism [8-10]. Thus, it is believed that PD NPs could adsorb lysosomal iron and cause an imbalance in the $\mathrm{Fe}^{2+} / \mathrm{Fe}^{3+}$ concentration that may propitiate an excessive generation of reactive oxygen species (ROS) through Fenton chemistry in cancer cells $[11,12]$.
This fact would explain why when iron chelators or antioxidant compounds are administered together with PD NPs the latter are not so cytotoxic and why the chelation of PD NPs with Fe increases their toxicity to tumor cells $[8,9,13]$. Moreover, as normal cells are not as dependent on iron as malignant cells $[14,15]$, the PD-mediated ferroptosis would not notably affect to their survival rate, as it has been already shown $[8,9]$.

On the other hand, it is relevant to mention that most of these findings related to the cytotoxicity of PD NPs have been performed by synthetizing them in a basic aqueous medium containing ethanol, in which the self-polymerization of dopamine occurs [8]. It has been reported that the use of alcohols in this chemical process can help to control PD NP size, which can range from tens to hundreds of nanometers by simply tuning the volume ratio of water to alcohol $[10,12]$. Among the different alcohols available, ethanol is the most frequently used possibly because it is completely miscible in water and is more polar than other types of alcohols [10,16]. Methanol and 2-propanol have also been employed to produce PD NPs [17]. However, the differences caused in PD NPs by the use of different alcohols in their synthesis have not been characterized and reported to date.

\footnotetext{
* Corresponding authors.

E-mail addresses: mvega@usal.es (M. Vega), emvalle@usal.es (E.M. Martín del Valle).
} 


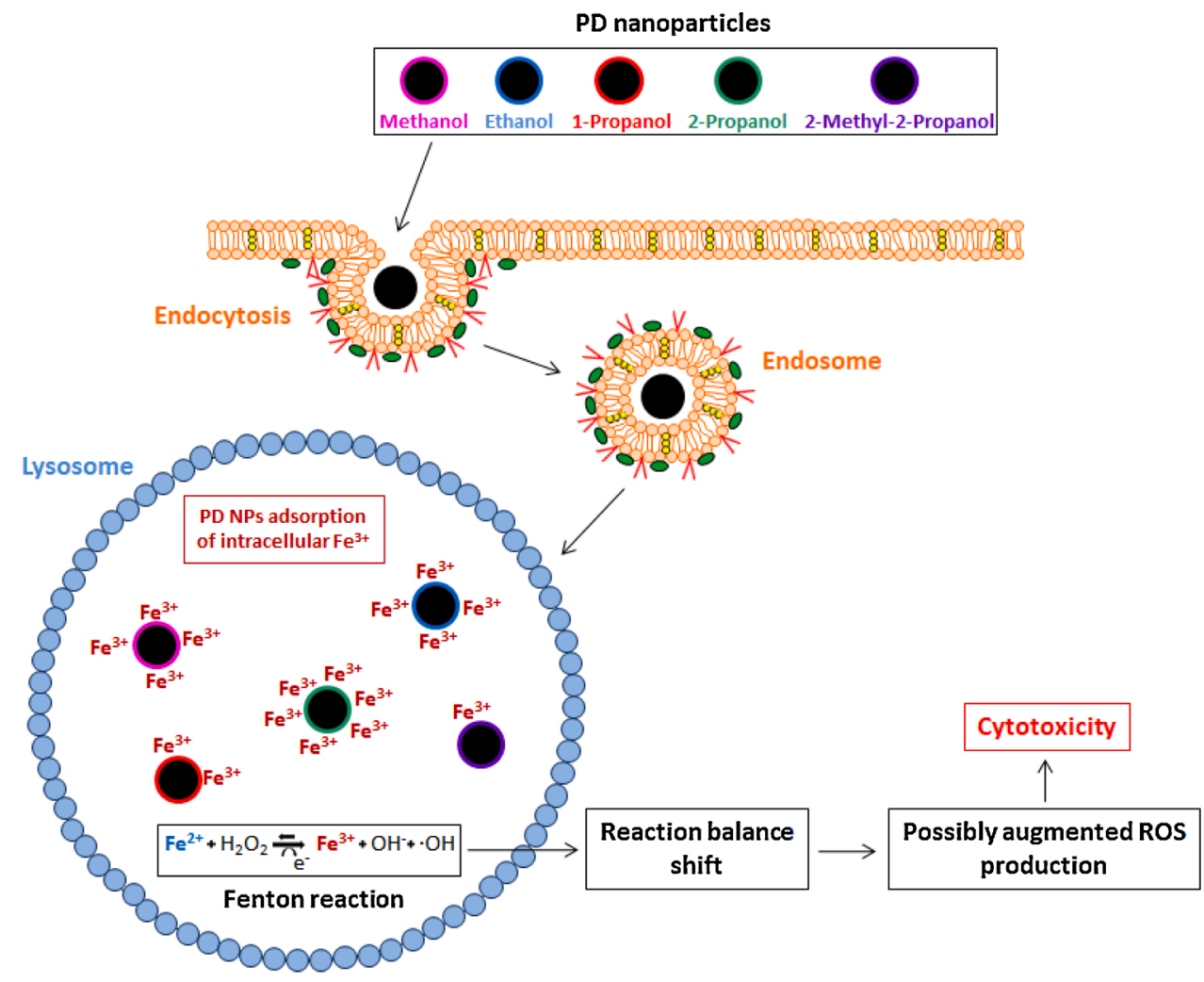

Fig. 1. Scheme representing how the type of ROH employed in the synthesis of PD NPs may determine their tumor-specific toxicity. Since the ROH used seems to condition the ability of PD NPs to adsorb $\mathrm{Fe}^{3+}$ cations, it may determine the production of ROS in treated cancer cells through the Fenton chemistry.

Therefore, in the present study, PD NPs have been synthesized in aqueous media containing water, ammonium hydroxide $\left(\mathrm{NH}_{4} \mathrm{OH}\right)$ and different alcohols (ROH), such as methanol (MeOH), ethanol (EtOH), 2propanol (2-PrOH), 1-propanol (1-PrOH) and 2-methyl-2-propanol (2$\mathrm{Me}-2-\mathrm{PrOH})$. The first aim was to analyze how the type of $\mathrm{ROH}$ and the water/ROH volume ratio employed, along with the concentration of $\mathrm{NH}_{4} \mathrm{OH}$ in the medium, influence particle size. In addition, since a ferroptosis-like mechanism may be responsible for PD NP toxicity to tumor cells, the $\mathrm{Fe}^{3+}$-loading capacity of the different $\mathrm{PD}(\mathrm{ROH}) \mathrm{NPs}$ produced has been also determined and compared. As a result, it has been shown that the type of ROH employed in PD NPs synthesis determines their $\mathrm{Fe}^{3+}$-adsorption capacity and, consequently, their cytotoxicity, which has been determined for a breast cancer cell line (Fig. 1). A correlation between both properties has been found and besides, it has been observed that such relationship is reverse for normal cells. Finally, with the co-administration of deferoxamine (DFO) and glutathione (GSH) (an iron chelator and an antioxidant compound, respectively) together with the PD NPs [11,12], it has been corroborated that Fenton chemistry may mediate the production of ROS in treated tumor cells.

\section{Materials and methods}

\subsection{Materials}

Dopamine hydrochloride, ammonium hydroxide, phosphate buffered saline (PBS, $0.01 \mathrm{M}, \mathrm{pH}$ 7.4), DMEM medium, Fetal Bovine Serum (FBS), MTT reagent, L-glutathione reduced (GSH) and deferoxamine mesilate (DFO) were provided by Sigma-Aldrich. Penicillin-streptomycin $(5000 \mathrm{U} / \mathrm{mL})$ was provided by Thermo Fisher Scientific. Iron (III) chloride anhydrous $\left(\mathrm{FeCl}_{3}, 97 \%\right.$ ), sodium acetate anhydrous, 1-propanol (extra pure), 2-propanol (99.5\%) and 2-methyl-2-propanol (99.5 $\%$ ) were provided by Panreac. Absolute ethanol (99.5 \%) and methanol (99.5 \%) were supplied by VWR Chemicals.

\subsection{Methods}

IR absorption spectra were performed in a PerkinElmer SpectrumTwo ${ }^{\mathrm{TM}}$ spectrometer operating in the $4000-400 \mathrm{~cm}^{-1}$ wavelength range after preparing samples as pellets of PD NPs in potassium bromide. TEM images were acquired with a FEI Tecnai Spirit Twin at an acceleration voltage of $120 \mathrm{kV}$. PD NPs were dispersed in deionized water in a concentration less than $0.01 \%$ (WT) and a drop of this dispersion was deposited on a copper grid with a collodion membrane and allowed to dry for $24 \mathrm{~h}$. Hydrodynamic diameter of PD NPs was analyzed by dynamic light scattering (DLS) on the basis of their intensity-average size distribution with the Zetasizer Nano ZS90. PD NPs were suspended in a Trizma buffer ( $50 \mathrm{mM}, \mathrm{pH} 10$ ) in a concentration less than $0.01 \%$ (WT) and their translational diffusion coefficient was calculated using the Stokes-Einstein equation (Eq. (1)), where $d(\mathrm{H})$ represents the hydrodynamic diameter, $D$ is the translational diffusion coefficient, $\mathrm{k}$ is the Boltzmann'z constant, $\mathrm{T}$ is absolute temperature and $\eta$ is viscosity. Correlation functions were analyzed by the Cumulants method

$d(H)=\frac{k T}{3 \pi \eta D}$

Finally, the equipment used for the $\mathrm{Fe}^{3+}$ analysis was the Plasma Emission Spectrometer, model ULTIMA 2 of Jobin Yvon. The calibration range used was $10-100 \mathrm{ppm}$.

\subsubsection{Synthesis of polydopamine nanoparticles (PD NPs)}

The synthesis of the different PD NPs was carried out in a water (90 $\mathrm{mL}) / \mathrm{ROH}(40 \mathrm{~mL})$ mixture, fixing the volume of the resulting solvent at $140 \mathrm{~mL}$ in all cases. $\mathrm{A} \mathrm{NH}_{4} \mathrm{OH}$ aqueous solution (28-30 \%) was added to the water/ROH mixtures under magnetic stirring at $25{ }^{\circ} \mathrm{C}$ for $30 \mathrm{~min}$, varying the concentration of $\mathrm{NH}_{4} \mathrm{OH}$ from 0.79 to $3.01 \%(\mathrm{~V} / \mathrm{V}$ ) depending on the type of ROH. Finally, dopamine hydrochloride $(0.5 \mathrm{~g})$ was dissolved in deionized water $(10 \mathrm{~mL})$ and directly added to the mixed solution. All polymerization reactions were allowed to proceed 
for $24 \mathrm{~h} \mathrm{[16].}$

Once obtained, $\mathrm{PD}(\mathrm{ROH}) \mathrm{NPs}$ were isolated by centrifugation and purified by at least four centrifugation-redispersion cycles in deionized water. In order to calculate dopamine conversion rate, the resulting products were oven-dried at $105^{\circ} \mathrm{C}$ until reaching a constant weight.

\subsection{2. $\mathrm{Fe}^{3+}$-loading capacity of the $P D(\mathrm{ROH}) \mathrm{NPS}$}

$\mathrm{Fe}^{3+}$-loaded PD(ROH) NPs were prepared by mixing approximately 1 $\mathrm{mL}$ of PD NPs with $20 \mathrm{~mL}$ of a $\mathrm{FeCl}_{3}$ solution (acetate buffer, $0.4 \mathrm{M}, \mathrm{pH}$ 4.5) at $25^{\circ} \mathrm{C}$, in orbital shaking at $100 \mathrm{rpm}$ overnight. Concentration and diameter (DLS) of NPs were: $\mathrm{MeOH}(2.4 \mathrm{mg} / \mathrm{mL}, 168 \mathrm{~nm} \pm 43$, PDI $=$ 0.026); EtOH (2.4 mg/mL, $169 \mathrm{~nm} \pm 37$, PDI = 0.026); 2-PrOH (2.8 mg/ $\mathrm{mL}, 187 \mathrm{~nm} \pm 37$, PDI $=0.055) ; 1$-PrOH $(2.7 \mathrm{mg} / \mathrm{mL}, 190 \mathrm{~nm} \pm 46$, PDI $=0.044)$; and 2-Me-2-PrOH $(3.9 \mathrm{mg} / \mathrm{mL}, 170 \pm 47$, PDI $=0.066)$. Different initial $\mathrm{Fe}^{3+}$ concentrations, from approximately 7-35 ppm, were studied for all PD(ROH) NPs. Then, loaded PD NPs were isolated by centrifugation and the $\mathrm{Fe}^{3+}$ content of the supernatant was determined by Inductively Coupled Plasma Atomic Emission Spectroscopy (ICPAES).

\subsection{3. $P D(R O H) N P s$ treatment for the cytotoxicity studies}

Isolated $\mathrm{PD}(\mathrm{ROH}) \mathrm{NPs}$ were purified by four centrifugationresuspension cycles in deionized water for carrying out the cytotoxicity studies. These PD NPs were later suspended in PBS (pH 7.4) with a final concentration and diameter (DLS) of approximately: i) $\mathrm{MeOH}(2.47$ $\mathrm{mg} / \mathrm{mL}, 173.0 \pm 51.3 \mathrm{~nm}, \mathrm{PDI}=0.067)$; ii) $\mathrm{EtOH}(1.76 \mathrm{mg} / \mathrm{mL}, 168.5 \pm$ $40.7 \mathrm{~nm}, \mathrm{PDI}=0.034)$; iii) 2 -PrOH $(2.8 \mathrm{mg} / \mathrm{mL}, 188.0 \pm 53.0 \mathrm{~nm}, \mathrm{PDI}=$ 0.047); iv)1-PrOH (1.50 mg/mL, $176.3 \pm 50.4 \mathrm{~nm}, \mathrm{PDI}=0.052)$; and v) 2-Me-2-PrOH (1.94 mg/mL, $170.8 \pm 53.7 \mathrm{~nm}, \mathrm{PDI}=0.077)$.

\subsubsection{Cell culture}

BT474 and HS5 cells were cultured at $37^{\circ} \mathrm{C}$ in a humidified atmosphere in the presence of carbon dioxide $\left(\mathrm{CO}_{2}\right)(5 \%)$. Culture medium (DMEM) was supplemented with FBS (10\%) and antibiotics (1\%) as instructed (ATCC, Wesel).

\subsubsection{Cytotoxicity studies}

To study the cytotoxicity of the PD(ROH) NPs, BT474 and HS5 were seeded in 24-well plates, with a density of 12,000 and 15,000 cells $/ \mathrm{mL}$ respectively, and grown overnight with supplemented medium. The culture medium was replaced the following day with medium containing PBS (control) and five different concentrations (from $0.0074 \mathrm{mg} / \mathrm{mL}$ to $0.042 \mathrm{mg} / \mathrm{mL}$ ) of the $\mathrm{PD}(\mathrm{ROH}) \mathrm{NPs}$. In all cases, the cellular survival rate was analyzed for $72 \mathrm{~h}$ (EZ Reader 2000), and cellular viability was checked every $24 \mathrm{~h}$ through MTT assays, following a previously described protocol [8]. Each value shown is the average of three independent experiments and the results obtained were considered statistically significant when $\mathrm{p}<0.05$.

\subsubsection{Effect of GSH and DFO on $P D(R O H)$ NPs cytotoxicity}

With the purpose of testing whether the capacity of PD NPs to adsorb $\mathrm{Fe}^{3+}$ could be involved in their antiproliferative activity, additional MTT assays were performed using the BT474 cell line, seeding them in 24well plates with at a density of 12,000 cells/mL $[5,8,9]$. After growing them overnight in supplemented medium, it was replaced the next day with medium containing: i) PBS (control); ii) DFO or GSH; iii) PD (MeOH) NPs; iv) PD(2-PrOH) NPs; v) PD(MeOH) NPs + DFO or GSH; and v) $\mathrm{PD}(2-\mathrm{PrOH}) \mathrm{NPs}+\mathrm{DFO}$ or GSH. In all cases, the concentrations for PD(ROH) NPs, DFO and GSH were $0.029 \mathrm{mg} / \mathrm{mL}, 0.7 \mu \mathrm{M}$ and $50 \mu \mathrm{M}$, respectively. The viability of the treated BT474 cells was studied again for $72 \mathrm{~h}$, and the cellular survival rates were quantified every $24 \mathrm{~h}$ using the previously mentioned protocol [8]. Each result shown is again the average value of the three independent experiments.
Table 1

Size (TEM, DLS) of the PD NPs, conversion yield and Ra values of dopamine polymerization reaction as a function of the $\mathrm{ROH}$ employed.

\begin{tabular}{|c|c|c|c|c|c|}
\hline $\mathrm{ROH}$ & $\varepsilon$ & $\begin{array}{l}\text { TEM } \\
(\mathrm{nm})\end{array}$ & DLS (nm) & $\begin{array}{l}\text { Yield } \\
\text { (\%) }\end{array}$ & $\mathrm{Ra}^{\mathrm{c}}$ \\
\hline $\mathrm{MeOH}$ & $33.1^{\mathrm{a}}$ & $382 \pm 26$ & $\begin{array}{l}628 \pm 145 \text { (PDI: } \\
0.229)\end{array}$ & 22.0 & 4.21 \\
\hline EtOH & $23.8^{\mathrm{a}}$ & $306 \pm 32$ & $\begin{array}{l}412 \pm 120 \text { (PDI: } \\
0.075)\end{array}$ & 17.8 & 3.06 \\
\hline 2-PrOH & $19.2^{\mathrm{b}}$ & $194 \pm 20$ & $282 \pm 70$ (PDI: 0.036$)$ & 14.6 & 2.82 \\
\hline 1-PrOH & $20.1^{\mathrm{b}}$ & $119 \pm 16$ & $191 \pm 41$ (PDI: 0.041) & 5.4 & 2.78 \\
\hline $\begin{array}{l}\text { 2-Me-2- } \\
\text { PrOH }\end{array}$ & $12.47^{\mathrm{b}}$ & $194 \pm 27$ & $286 \pm 70$ (PDI: 0.206$)$ & 13.0 & 3.13 \\
\hline
\end{tabular}

\section{Results and discussion}

\subsection{Effect of the different types of alcohols on the synthesis of PD NPs}

PD NPs were prepared using the standard procedure of oxidative polymerization of dopamine in a basic aqueous medium containing $\mathrm{NH}_{4} \mathrm{OH}$ and $\mathrm{ROH}$. In order to study the effect that the type of $\mathrm{ROH}$ had on this chemical process, NP synthesis was performed with five different ROHs of 1, 2, 3 and 4 carbons. In all cases, the water/ROH volume ratio $(10: 4,28.57 \%(\mathrm{~V} / \mathrm{V}))$ and the $\mathrm{NH}_{4} \mathrm{OH}$ concentration $(0.79 \% \mathrm{~V} / \mathrm{V})$ were kept constant. Resulting PD(ROH) NPs were characterized by DLS and TEM and the values of the Hansen's Solubility Parameters (HSP) distances (Ra values) between dopamine and all the water/ROHs mixtures were determined (Table 1 ).

As can be seen, $\mathrm{MeOH}$ produced the largest NPs, followed by those produced using EtOH, 2-PrOH, 2-Me-2-PrOH and, finally, 1-PrOH. PD (ROH) NPs size, as determined by TEM, ranged from $382 \pm 26 \mathrm{~nm}$ $(\mathrm{MeOH})$ to $120 \pm 16 \mathrm{~nm}$ (1-PrOH), which indicated that the higher the alcohol dielectric constant $(\varepsilon)$, the greater the NP diameter and the polydopamine conversion yield. By contrast, PD NPs produced with 1$\mathrm{PrOH}$ were smaller despite 1-PrOH having a high $\varepsilon$ (20.1) [18]. The values for the hydrodynamic diameter obtained by DLS were higher than those obtained by TEM possibly due to the dehydration of the NPs that was necessary to perform to prepare the samples for microscopy, but followed the same trend in both cases [19]. Moreover, obtained NP polydispersity index (PDI) values were acceptable, and those lower than 0.1 indicated that the PD(ROH) NPs were highly monodisperse [20].

According to the literature, the type of $\mathrm{ROH}$ used affects dopamine polymerization, being this effect explained by the HSP theory, in which Ra values refer to the three-dimensional distance between the solubility parameters of dopamine and the solvent (water/ROH mixtures) [18]. Thus, Jiang et al. had previously demonstrated that there is a relationship between dopamine conversion yield and PD NP size, in turn conditioned by the water/ROH volume ratio employed. These authors calculated the Ra values among dopamine and three different water/$\mathrm{ROH}$ (EtOH, $\mathrm{MeOH}$ and 2-PrOH) solvent mixtures and reported that the smaller the Ra value, the higher the expected dopamine solubility and the higher the polymer conversion yield achieved. In addition, they also concluded that PD NPs can be more easily obtained in weakly alkaline water/ROH solvent mixtures with smaller Ra values [18].

In this way, the Ra values determined between dopamine and the five ROHs tested in this work showed that the water/ROH volume ratio $(10: 4,28.57 \%(V / V))$ used was suitable for synthetizing PD NPs with $\mathrm{MeOH}, \mathrm{EtOH}$ and 2-PrOH because the obtained values were close to the minimum Ra values reported by Jiang et al. [18]. However, when 1-PrOH was used, the Ra value determined in this work (2.78) for the mentioned water/ROH ratio was far from being optimum. The optimal Ra value $(8: 6,42.86 \%(\mathrm{~V} / \mathrm{V}))$, with which a $297.5 \pm 62.3 \mathrm{~nm}$ particle 


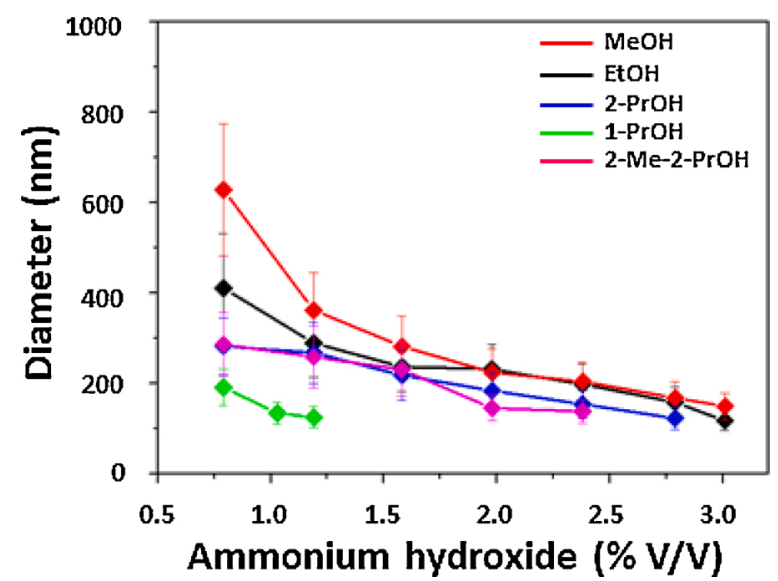

Fig. 2. Hydrodynamic diameter (nm) of the $\mathrm{PD}(\mathrm{ROH}) \mathrm{NPs}$ as a function of the $\mathrm{NH}_{4} \mathrm{OH}$ concentration used in their synthesis with each water/ROH mixture.

size was obtained, was found by varying the proportion of $\mathrm{ROH}$ in the solvent mixture from $35.71 \%(\mathrm{~V} / \mathrm{V})$ to $50.0 \%(\mathrm{~V} / \mathrm{V})$. Finally, regarding the use of 2-Me-2-PrOH, the Ra value obtained in this work $(\mathrm{Ra}=3.13$; $28.57 \%(\mathrm{~V} / \mathrm{V})$ ) was also appropriate, since lower and higher $\mathrm{ROH}$ volume ratios (from $14.28 \%$ to $21.43 \%$ and $35.71 \%(\mathrm{~V} / \mathrm{V})$ ) led to obtain higher Ra values. All the steps taken in this work to determine the described Ra values can be consulted in the Supplementary Material (Tables S1-S4).

Otherwise, in a second experiment, the effect of the $\mathrm{NH}_{4} \mathrm{OH}$ concentration on $\mathrm{PD}(\mathrm{ROH})$ NPs size was also studied. Fig. 2 and S1 show that for a fixed water/ROH volume ratio, the size of PD NPs could be altered by varying the concentration of $\mathrm{NH}_{4} \mathrm{OH}$, whose main function in the synthesis process is to create a basic medium that allows polydopamine to be oxidized.

In general, for all of the $\mathrm{PD}(\mathrm{ROH})$ systems studied, lower diameters were obtained by increasing the concentration of $\mathrm{NH}_{4} \mathrm{OH}$, since the $\mathrm{pH}$ of the synthesis medium conditions PD oxidation and polymerization, as it had been previously demonstrated $[19,21]$. When the $\mathrm{NH}_{4} \mathrm{OH}$ concentration was set at $0.79 \%(\mathrm{~V} / \mathrm{V})$, the smallest PD NPs $(191 \mathrm{~nm})$ were obtained using 1-PrOH and the largest $(628 \mathrm{~nm})$ using $\mathrm{MeOH}$. Also, it was interesting to find that for 1-PrOH, the diameter of the NPs varied by $290 \mathrm{~nm}$ when $\mathrm{NH}_{4} \mathrm{OH}$ concentration was increased from 0.79 to $1.19 \%$ (V/V) and by $65 \mathrm{~nm}$ when the concentration increased from 1.19 to 1.58 $\%(\mathrm{~V} / \mathrm{V})$. A similar behavior in size was found, based on the $\mathrm{NH}_{4} \mathrm{OH}$ concentration, for PD NPs obtained with $\mathrm{MeOH}$ and EtOH. The difference in their diameter when the $\mathrm{NH}_{4} \mathrm{OH}$ concentration was increased from 1.19 to $1.58 \%(\mathrm{~V} / \mathrm{V}$ ) was 260 and $120 \mathrm{~nm}$, respectively. However, when larger amounts of $\mathrm{NH}_{4} \mathrm{OH}$ were used, such difference was not so marked, similar to what was observed when 2-PrOH and 2-Me-2-PrOH were employed. Data corresponding to the $\mathrm{PD}(\mathrm{ROH}) \mathrm{NPs}$ diameter values as a function of the $\mathrm{NH}_{4} \mathrm{OH}$ concentration employed can be found in the Supplementary Material, too (Fig. S1F).

Finally, in order to obtain PD(ROH) NPs smaller than $100 \mathrm{~nm}$, which are interesting from a biomedical perspective [12,22], higher concentrations of $\mathrm{NH}_{4} \mathrm{OH}$ were tested. Fig. 3A shows the size of the PD NPs produced according to the type of $\mathrm{ROH}$ used and Fig. 3B-F shows the morphology of these PD(ROH) NPs smaller than $100 \mathrm{~nm}$.

\subsection{Infrared characterization of $P D(R O H) N P S$}

IR spectroscopy has been widely used to characterize melanins $[5,23$, 24]. Thus, main bands were here assigned according to the previous characterization of melanins and were the following: the broad absorption band with the maximum at $1612 \mathrm{~cm}^{-1}$ may include the $\mathrm{C}=\mathrm{O}$ stretching (non-carboxylic acid) and the $\mathrm{C}=\mathrm{C}$ stretching of the aromatic system (at ca $1580 \mathrm{~cm}^{-1}$ ). The $\mathrm{N}-\mathrm{H}$ bending in an indole ring may
A

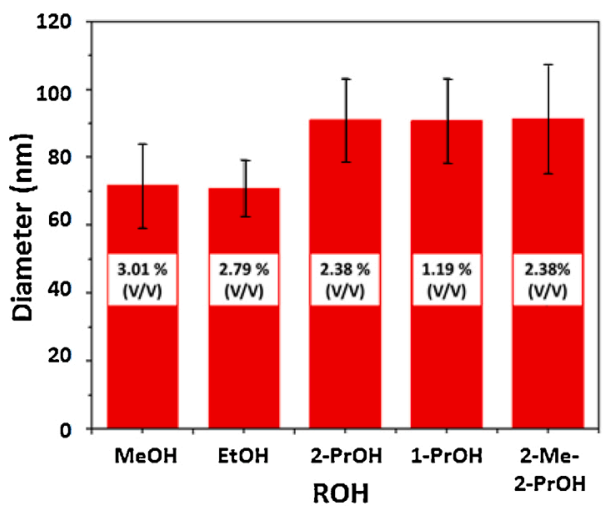

D

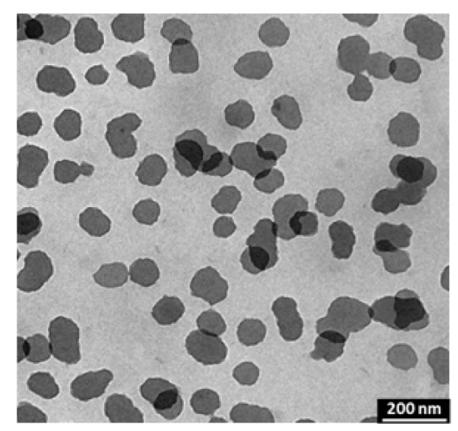

B

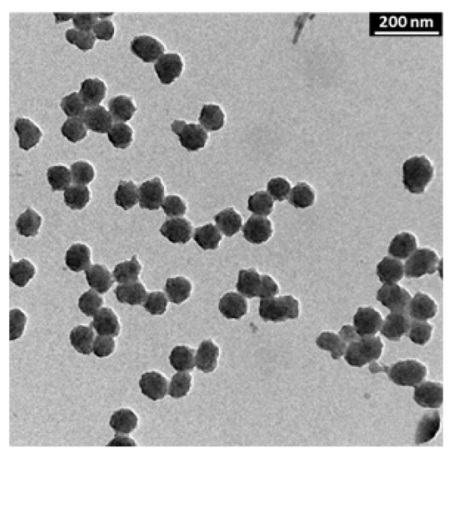

E

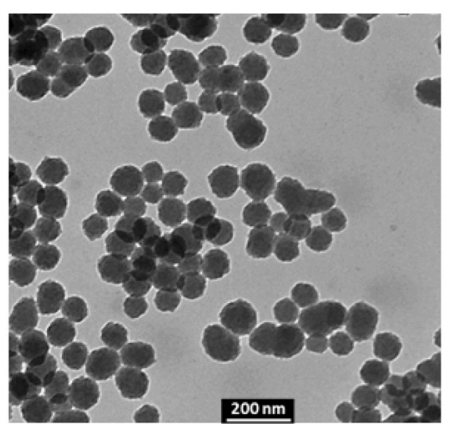

C

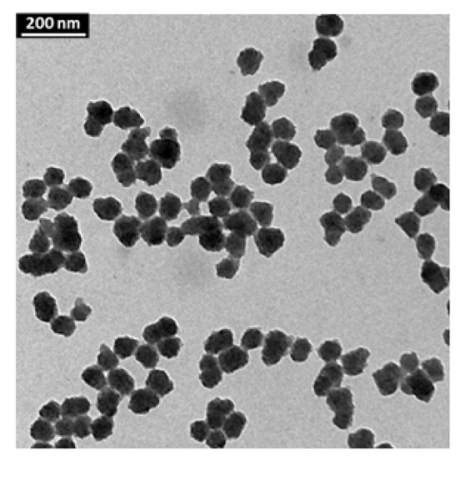

F

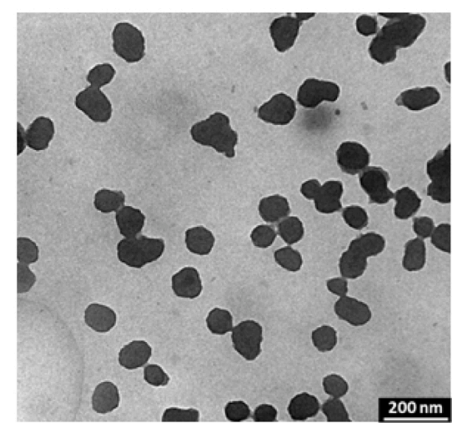

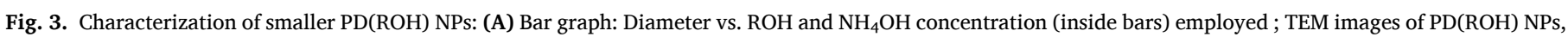

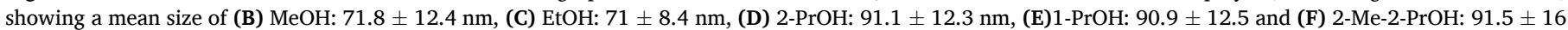
$\mathrm{nm}$. Scale bars are $200 \mathrm{~nm}$ in all cases. 
A

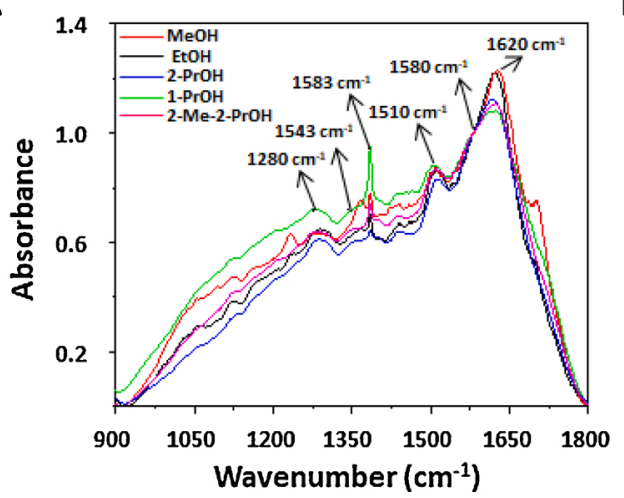

B

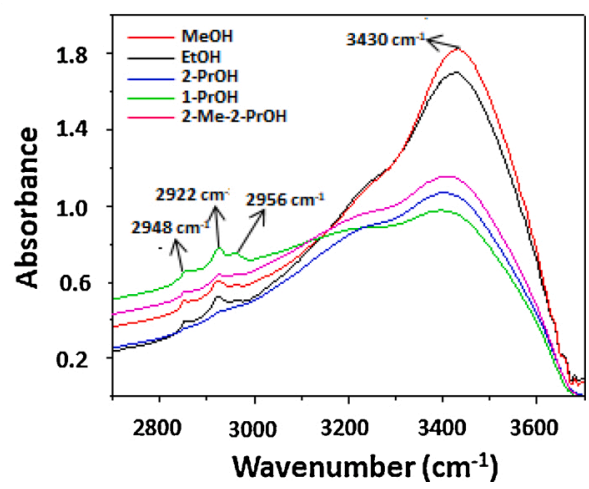

Fig. 4. IR spectra in the $900-1800 \mathrm{~cm}^{-1}$ range (A) and in the $2700-3700 \mathrm{~cm}^{-1}$ range (B) for all the PD(ROH) NPs.

A

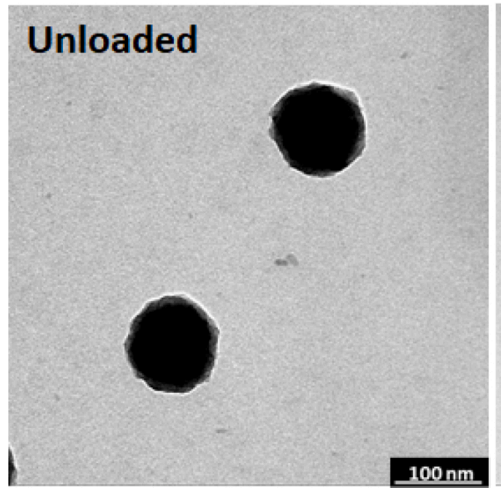

B

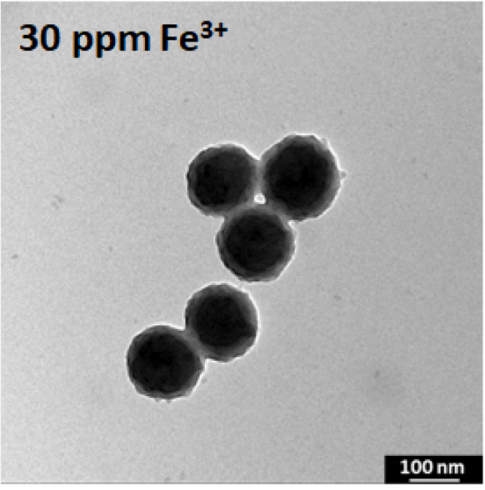

C

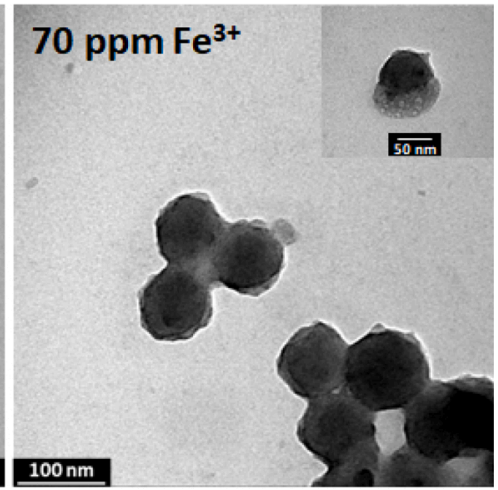

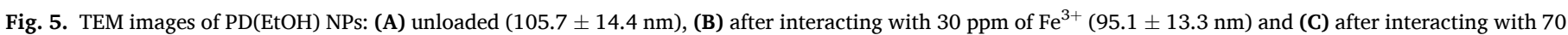
ppm of $\mathrm{Fe}^{3+}(97.8 \pm 14.0 \mathrm{~nm})$. Scale bars are $100 \mathrm{~nm}$.

appear at $1510 \mathrm{~cm}^{-1}$, while the peaks at 1345 and $1383 \mathrm{~cm}^{-1}$ may correspond to the $\mathrm{C}-\mathrm{N}$ stretching mode of an indole and a pyrrole ring, respectively. The $\mathrm{C}-\mathrm{OH}$ stretching in a phenol ring appears at 1280 $\mathrm{cm}^{-1}$. In the region from 4000 to $2000 \mathrm{~cm}^{-1}$, the bands at 2956,2922 and $2840 \mathrm{~cm}^{-1}$ correspond to the different vibrational modes of the aliphatic groups. The broad band placed at $3405 \mathrm{~cm}^{-1}$ may include the $-\mathrm{OH}$ (phenol, carboxylic acid) and the - $\mathrm{NH}$ stretching modes (in an indole or in a primary amine).

IR spectra of PD NPs obtained in the different water/ROH media are shown in Fig. 4. Spectra were normalized at $1580 \mathrm{~cm}^{-1}$ in order to compare the intensity of the different bands.

The above-described characteristic bands of melanins within the $900-1800 \mathrm{~cm}^{-1}$ range can be seen in all PD(ROH) NPs. This is also the case within the $2700-3700 \mathrm{~cm}^{-1}$ range, but the relative intensity of the band at $3430 \mathrm{~cm}^{-1}$ associated with $-\mathrm{OH}$ and $-\mathrm{NH}$ stretching modes notably decreased when either 1-PrOH, 2-PrOH or 2-Me-2-PrOH were used for PD NPs synthesis.

\subsection{Influence of the use of different alcohols on PD NPs $\mathrm{Fe}^{3{ }^{+}}$-adsorption capacity}

Here, the $\mathrm{Fe}^{3+}$-adsorption capacity of the PD(ROH) NPs was studied. First, changes in the morphology of $\mathrm{PD}(\mathrm{EtOH}) \mathrm{NPs}$ upon interaction with two initial $\mathrm{Fe}^{3+}$ concentrations ( 30 and $70 \mathrm{ppm}$ ) were evaluated. TEM image in Fig. 5A shows a control of unloaded PD NPs, while the slight variation in the morphology of $\mathrm{Fe}^{3+}$-loaded NPs can be observed in Fig. $5 \mathrm{~B}-\mathrm{C}$, especially when the initial $\mathrm{Fe}^{3+}$ concentration was higher, since the NPs seem to be spongier.

Then, $\mathrm{PD}(\mathrm{ROH}) \mathrm{NP} \mathrm{Fe}^{3+}$-loading capacity was further analyzed, fitting the equilibrium results using the Freundlich isotherm, which is applied in multilayer adsorption processes on heterogeneous surfaces $[25,26]$. The initial $\mathrm{Fe}^{3+}$ concentration added varied in the range of 7-35 ppm at pH 4.5, while the concentration of the PD NPs was kept constant to allow for proper comparisons. TEM images of the NPs used to obtain the isotherms can be found in the Supplementary Material (Fig. S2). As a result, it was shown that all $\mathrm{PD}(\mathrm{ROH}) \mathrm{NPs}$ were able to adsorb this metal cation, which made their surface spongier; a fact that was in accordance with what had already been reported in the literature [11]. Nevertheless, according to the equilibrium results, $\mathrm{Fe}^{3+}$-loading capacity varied depending on the water/ $\mathrm{ROH}$ synthesis mixture employed. This difference was more accentuated when a higher $\mathrm{Fe}^{3+}$ concentration (35 ppm) was used, while the difference in the adsorption capacity of the $\mathrm{PD}(\mathrm{ROH})$ NPs was negligible for a lower concentration (7 ppm). PD NPs prepared in 2-PrOH showed the highest absorption capacity of $\mathrm{Fe}^{3+}$ with respect to the other $\mathrm{PD}(\mathrm{ROH})$ systems and, by contrast, $\mathrm{PD}(2-\mathrm{Me}-2-\mathrm{PrOH}) \mathrm{NPs}$ were clearly the NPs with the lowest $\mathrm{Fe}^{3+}$-adsorption capacity. Linear fittings according to Freundlich model, $\mathrm{Fe}^{3+}$-adsorption efficiency (\%) and fitted parameters $(1 / n$ and $Q)$ can also be consulted in the Supplementary Material (Fig. S3 and Table S4).

\subsection{Cytotoxicity of the different $P D(R O H) N P S$}

In this section, the cytotoxicity of the $\mathrm{PD}(\mathrm{ROH}) \mathrm{NPs}$ synthetized was analyzed. Previously, it had been reported that standard PD(EtOH) NPs were intrinsically toxic to cancer cells, especially to the breast carcinoma cell line BT474 $[5,8,9]$. For this reason, this human cell line was chosen to study the antitumor effect of the different PD(ROH) NPs produced in this work. Likewise, the stromal HS5 cell line was selected 
A

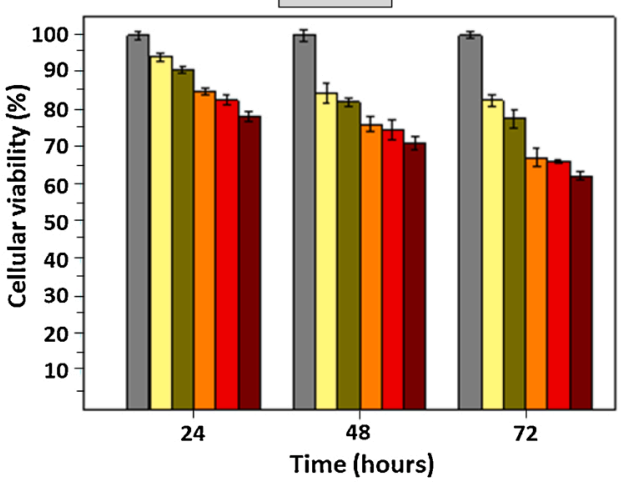

C

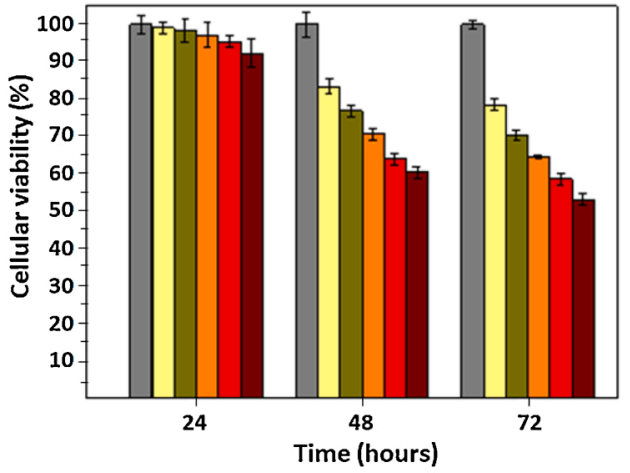

B

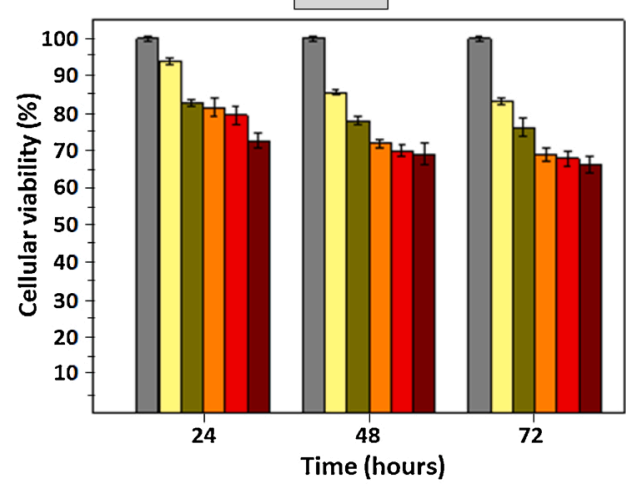

D

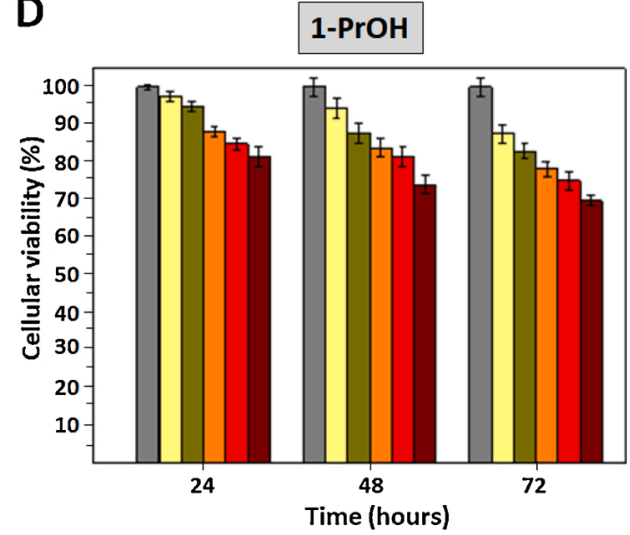

E 2-Me-2-PrOH

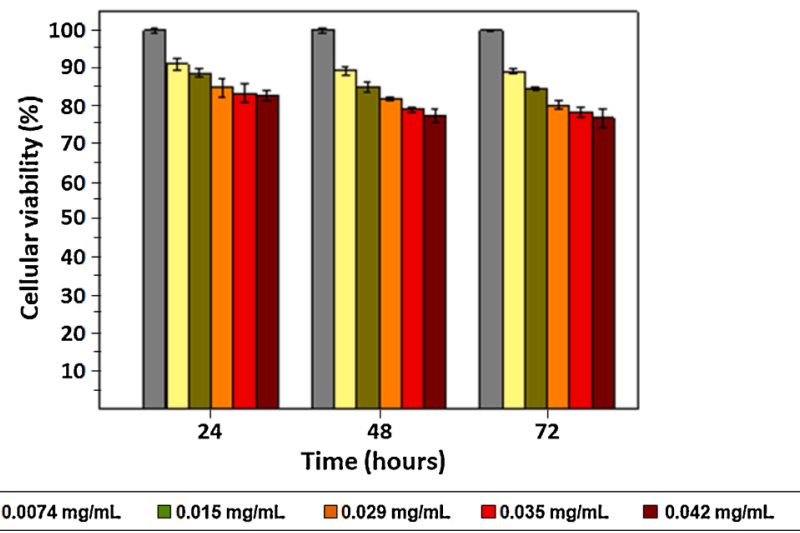

Fig. 6. Results of the MTT assay performed on the BT474 cell line treated with five different concentrations of PD(ROH) $\mathrm{NPs}$. ROH $=(\mathrm{A}) \mathrm{MeOH}$, (B) EtOH, (C) 2 $\mathrm{PrOH}$, (D) 1-PrOH and (E) 2-Me-2-PrOH. The results shown represent the mean \pm standard deviation of three replicas done for each treatment.

for comparing $\mathrm{PD}(\mathrm{ROH}) \mathrm{NPs}$ toxicity to tumor and normal cells. The results of the viability assays carried out on both cell lines, treated with different concentrations of the $\mathrm{PD}(\mathrm{ROH}) \mathrm{NPs}$, can be found in Fig. 6 (BT474 cells) and in Fig. 7 (HS5 cells). Additionally, a table with the survival rates (\%) corresponding to these figures can be found in the Supplementary Material (Fig. S5).

As shown in Fig. 6, the PD(ROH) NPs caused, in all cases, a notable reduction in the viability of BT474 cells. This result was especially apparent $72 \mathrm{~h}$ after the cells were treated with the highest concentrations of NPs $(0.035 \mathrm{mg} / \mathrm{mL}$ and $0.042 \mathrm{mg} / \mathrm{mL})$. Among the different types of PD NPs, those synthesized using $\mathrm{MeOH}, \mathrm{EtOH}$ and 1-PrOH exhibited practically the same level of cytotoxicity. It was observed that these PD NPs were able to reduce the rate of cell survival by $20-25$ $\% 24 \mathrm{~h}$ after treatment with $\mathrm{NP}$ concentrations of $0.035 \mathrm{mg} / \mathrm{mL}$ and $0.042 \mathrm{mg} / \mathrm{mL}$. However, the survival rate of these cells was slightly higher when they were treated with the same concentrations of $\mathrm{PD}(2$ Me-2-PrOH) NPs, and even more when $\mathrm{PD}(2-\mathrm{PrOH})$ NPs were administered. Nevertheless, this pattern changed once 48 and $72 \mathrm{~h}$ elapsed. After such time, the highest concentrations of $\mathrm{PD}(\mathrm{MeOH}), \mathrm{PD}(\mathrm{EtOH})$ and $\mathrm{PD}$ (1-PrOH) NPs used reduced cellular viability by around $30-40 \%$, while cell treatment with $\mathrm{PD}(2-\mathrm{Me}-2-\mathrm{PrOH})$ NPs caused a viability reduction of $25-30 \%$. However, the cytotoxicity of the $\mathrm{PD}(2-\mathrm{PrOH}) \mathrm{NPs}$ was more marked, where BT474 cell survival rate was reduced to approximately half. In addition, it was observed that for the other PD (ROH) NP concentrations, the behavior was similar to that described for the highest concentrations, although their antitumor effect was not as marked.

Also, no significant reduction in HS5 cell viability was found for any of the PD(ROH) NPs (Fig. 7). In all cases, the rates of cellular survival were higher than $82 \% 72 \mathrm{~h}$ after treatment with the highest NP 
A

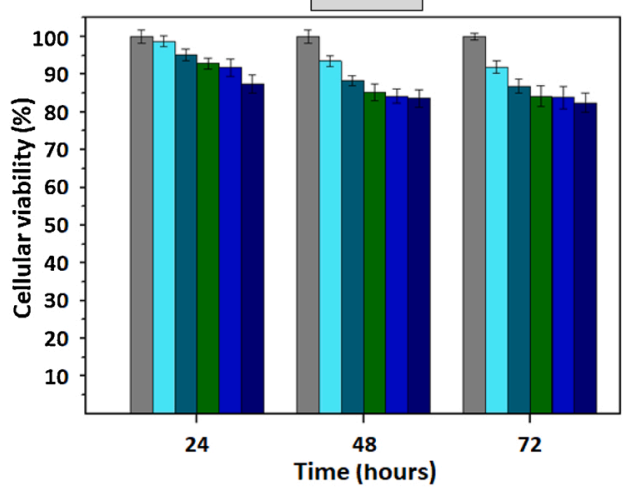

C

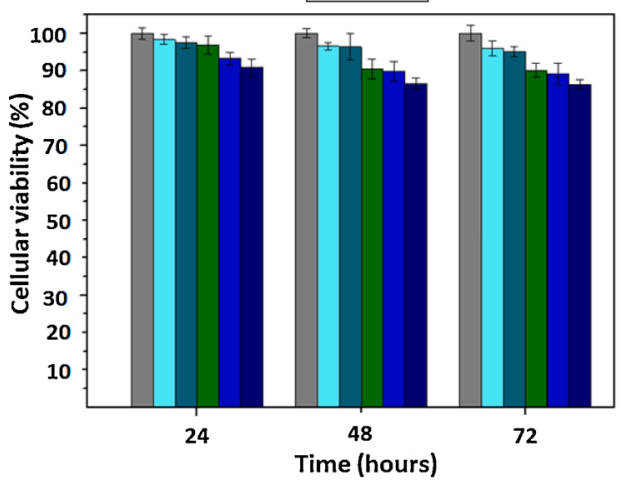

B

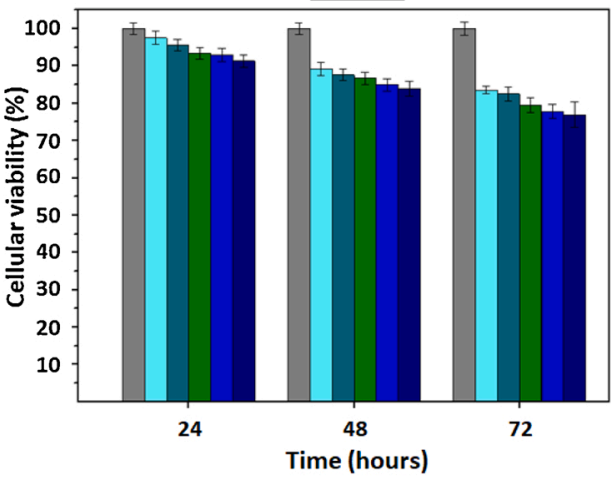

D 1 -PrOH

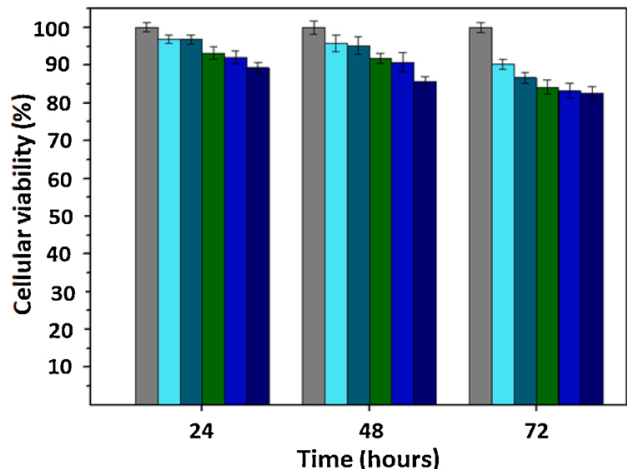

E 2-Me-2-PrOH

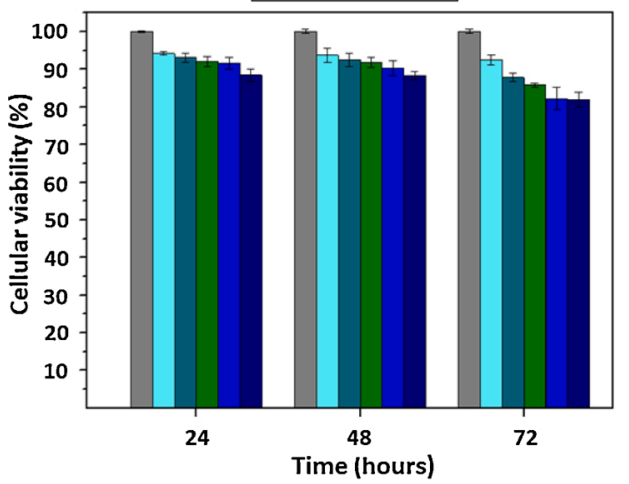

$\square$ Control $\square 0.0074 \mathrm{mg} / \mathrm{mL} \square 0.015 \mathrm{mg} / \mathrm{mL} \square 0.029 \mathrm{mg} / \mathrm{mL} \square 0.035 \mathrm{mg} / \mathrm{mL} \square 0.042 \mathrm{mg} / \mathrm{mL}$

Fig. 7. Results of the MTT assays performed on the HS5 stromal cell line treated with five different concentrations of (A) PD(MeOH), (B) PD(EtOH), (C) PD(2-PrOH), (D) PD(1-PrOH) and (E) PD(2-Me-2-PrOH) NPs. The results shown represent the mean \pm standard deviation of the three replicas done for each treatment.

concentrations, except when treatment was performed with $\mathrm{PD}$ (EtOH) NPs, which were the most toxic to stromal cells. When comparing the viability percentages of both BT474 and HS5 cell lines, treated with the same concentrations of NPs, greater differences were found after $72 \mathrm{~h}$, the time point when PD NPs antitumor activity was the highest. Taking into account the results obtained, it should be mentioned that the less selective NPs were the ones prepared in 2-Me-2-PrOH, while the NPs with the most selective antitumor activity were the $\mathrm{PD}(2-\mathrm{PrOH}) \mathrm{NPs}$.

As shown, PD(2-PrOH) NPs were the NPs that decreased BT474 cellular viability the most (to $53 \%$ after $72 \mathrm{~h}$ of the treatment with a $0.042 \mathrm{mg} / \mathrm{L}$ concentration) and had the most noticeable $\mathrm{Fe}^{3+}$-adsorption capacity $(140 \mathrm{mg} / \mathrm{g}$ for $\mathrm{Ci}=35 \mathrm{ppm})$. Conversely, $\mathrm{PD}(2-\mathrm{Me}-2-\mathrm{PrOH}) \mathrm{NPs}$ exhibited the lowest $\mathrm{Fe}^{3+}$-adsorption efficiency and had the lowest ability to reduce the survival rate of the BT474 cells (reduced to $73 \%$ after $72 \mathrm{~h}$ of treatment with a the highest concentration). This relation between $\mathrm{Fe}^{3+}$-loading and BT474 cell toxicity was also similar for all of the other $\mathrm{PD}(\mathrm{ROH}) \mathrm{NPs}$, except for the $\mathrm{PD}(\mathrm{EtOH}) \mathrm{NPs}$, with which similar results were obtained to those that had already been reported [8, 9]. As a result, a linear correlation could be established between both properties (Fig. S6). Also, this relation seemed to be inverse for HS5 cells, where a lower $\mathrm{Fe}^{3+}$-adsorption capacity of PD NPs was accompanied by a higher toxicity to stromal cells.

Iron is an essential element for most organisms, with a fundamental role for cell growth and proliferation. However, as mentioned in the introduction, iron can also be implicated in the generation of reactive oxygen species (ROS) through its participation in Fenton's chemistry, triggering cell death $[11,15,27]$. For this reason, it was believed that PD $\mathrm{Fe}^{3+}$-adsorption capacity could be related to $\mathrm{PD}(\mathrm{ROH}) \mathrm{NPs}$ tumor-specific toxicity and to verify this fact, additional viability assays were carried out, where breast cancer cells were co-treated with DFO or 
GSH.

\subsection{Antiproliferative effect of $P D(R O H)$ NPs on cancer cells Co-treated with DFO or GHS}

In order to show that the $\mathrm{Fe}^{3+}$-adsorption capacity of PD(ROH) NPs could be involved in their tumor-selective toxicity, BT474 cells were cotreated with $\mathrm{PD}(\mathrm{MeOH}, \mathrm{EtOH}$ and 2-PrOH) NPs and with non-toxic concentrations of DFO and GHS. These three types of $\mathrm{PD}(\mathrm{ROH})$ NPs were the ones selected to perform further viability assays because they presented greater anticancer activity. Also, DFO and GHS were chosen because they are an iron-chelating compound and an antioxidant agent, respectively $[28,29]$. Thus, additional MTT assays were carried out, studying BT474 cellular viability 24,48 and $72 \mathrm{~h}$ after the aforementioned co-treatment [8]. As shown in Fig. S7, it could be seen that such co-treatment managed to partially block the antiproliferative activity of $\mathrm{PD}(\mathrm{ROH}) \mathrm{NPs}$, since the cellular survival rate was on average higher than $80 \%$. Major differences between the cytotoxicity of the treatment with $\mathrm{PD}(\mathrm{ROH}) \mathrm{NPs}$ or with $\mathrm{PD}(\mathrm{ROH}) \mathrm{NPs}$ plus DFO or GSH could again be noticed after $72 \mathrm{~h}$ and were greater when BT474 cells were treated with $\mathrm{PD}(2-\mathrm{PrOH}) \mathrm{NPs}$.

Consequently, it was found that the $\mathrm{Fe}^{3+}$-loading of the PD NPs could account for their antitumor effect. Thus, when these NPs are internalized and end up in cellular lysosomes [8], they could cause an imbalance between the oxidized and reduced free iron forms and mediate an enhanced production of ROS through the Fenton reaction. In addition, since cancer cells have a greater iron-dependence than normal cells [14, $15]$, the viability of the latter was not so reduced by the PD NPs cytotoxicity mediated by their $\mathrm{Fe}^{3+}$-loading capacity.

\section{Conclusions}

Throughout the present study it was shown that $\mathrm{MeOH}$, EtOH, 2PrOH, 1-PrOH and 2-Me-2-PrOH can all be used to synthetize PD NPs, which size depended on the type of ROH used. Generally, the lower the $\varepsilon$ of the $\mathrm{ROH}$, the smaller the PD NP diameter, which also depended on the $\mathrm{NH}_{4} \mathrm{OH}$ concentration used in the synthesis process. With regard to the latter, the higher the concentration of $\mathrm{NH}_{4} \mathrm{OH}$ added, the smaller the size of the NPs produced.

In addition, it has also been proven that $\mathrm{Fe}^{3+}$-loading capacity of $\mathrm{PD}$ NPs was determined by the ROH used in their synthesis. Since a ferroptosis-like mechanism may account for PD NP cytotoxicity, the $\mathrm{ROH}$ used may also influence the final outcome. Cancer cells present great dependence on iron and, for this reason, PD NPs that are more efficient at adsorbing $\mathrm{Fe}^{3+}$ may cause a greater imbalance in the concentration of their lysosomal $\mathrm{Fe}^{2+}$ and $\mathrm{Fe}^{3+}$ when they are endocyted and propitiate an increase in ROS production. This fact would help to explain why $\mathrm{PD}(2-\mathrm{PrOH}) \mathrm{NPs}$, the ones with a higher $\mathrm{Fe}^{3+}$-adsorption capacity, were the NPs that most reduced the survival rate of breast cancer cells. Finally, this fact could also explain why PD NP toxicity was partially reduced when cells were co-treated with an iron chelator or an antioxidant compound. Moreover, since non-tumor cells are not that iron-dependent, the ferroptosis-like mechanism mediated through PD NPs may shed light on why such NPs did not significantly reduce their viability.

Therefore, based on the results presented here, it is clear that PD NPs, especially those prepared using 2-PrOH, may play an important role in the development of future and novel cancer therapy strategies.

\section{CRediT authorship contribution statement}

Celia Nieto: Conceptualization, Methodology, Writing - original draft, Writing - review \& editing. Gema Marcelo: Conceptualization, Methodology, Writing - review \& editing. Milena Vega: Conceptualization, Methodology, Supervision, Writing - original draft, Writing review \& editing. Eva M. Martín del Valle: Conceptualization, Writing - review \& editing, Funding acquisition.

\section{Declaration of Competing Interest}

The authors declare that they have no known competing financial interests or personal relationships that could have appeared to influence the work reported in this paper.

\section{Acknowledgments}

This research was funded by the Spanish Ministry of Economy and Competitiveness (grant number CTQ2016-78988-R) and by the Ramón Areces Foundation. C. Nieto thanks Junta de Castilla y Leon for the predoc fellowships. The authors thank the Microscopy Service of the University of Salamanca for the TEM images.

\section{Appendix A. Supplementary data}

Supplementary material related to this article can be found, in the online version, at doi:https://doi.org/10.1016/j.colsurfb.2020.111506.

\section{References}

[1] H. Lee, S.M. Dellatore, W.M. Miller, P.B. Messersmith, Mussel-inspired surface chemistry for multifunctional coatings, Science 318 (2007) 426-430.

[2] R. Mrówczynski, Polydopamine-based multifunctional (nano)materials for cancer therapy, ACS Appl. Mater. Interfaces 10 (2018) 7541-7561.

[3] P. Meredith, B.J. Powell, J. Riesz, S.P. Nighswander-Rempel, M.R. Pederson, E. G. Moored, Towards structure-property-function relationships for eumelanin, Soft Matter 2 (2006) 37-44.

[4] J.B. Nofsinger, E.E. Weinert, J.D. Simon, Establishing structure-function relationships for eumelanin, Biopolymers 67 (2002) 302-305.

[5] M.A. Vega, C. Nieto, G. Marcelo, E.M. Martín del Valle, Cytotoxicity of paramagnetic cations_Loaded polydopamine nanoparticles, Colloid Surf. B 167 (2018) 284-290.

[6] K.C.L. Black, J. Yi, J.G. Rivera, D.C. Zelasko-Leon, P.B. Messersmith, Polydopamine-enabled surface functionalization of gold nanorods for cancer celltargeting imaging and photothermal therapy, Nanomedicine 8 (2012) 17-28.

[7] Y. Li, C. Jiang, D. Zhang, Y. Wang, X. Ren, K. Ai, X. Chen, L. Lu, Targeted polydopamine nanoparticles enable photoacoustic imaging guide chemophotothermal synergist therapy of tumor, Acta Biomater. 47 (2017) 124-134.

[8] C. Nieto, M.A. Vega, G. Marcelo, E.M. Martín del Valle, Polydopamine nanoparticles kill cancer cells, RSC Adv. 8 (2018) 36201-36208.

[9] C. Nieto, M.A. Vega, J. Enrique, G. Marcelo, E.M. Martín del Valle, Size matters in the cytotoxicity of polydopamine nanoparticles in different types of tumors, Cancers 11 (2019) 1679.

[10] K.Y. Ju, Y. Lee, S. Lee, S.B. Park, J.K. Lee, Bioinspired polymerization of dopamine to generate melanin-like nanoparticles having an excellent free-radical-scavenging property, Biomacromolecules 12 (2011) 625-632.

[11] S.J. Dixon, B.R. Stockwell, The role of iron and reactive oxygen species in cell death, Nat. Chem. Biol. 10 (2014) 9-17.

[12] V. Corcé, S.G. Gouin, S. Renaud, F. Gaboriau, D. Deniaud, Recent advances in cancer treatment by iron chelators, Bioorg. Med. Chem. Lett. 26 (2016) 251-256.

[13] L. Chen, Z. Lin, L. Liu, X. Zhang, W. Shi, D. Ge, Y. Sun, $\mathrm{Fe}^{2+} / \mathrm{Fe}^{3+}$ ions chelated with ultrasmall polydopamine nanoparticles induce ferroptosis for cancer therapy, ACS Biomater. Sci. Eng. 5 (2019) 4861-4869.

[14] D.H. Manz, N.L. Blanchette, B.T. Paul, F.M. Torti, S.V. Torti, Iron and cancer: recent insights, Ann. NY Acad. Sci. 1368 (2016) 149-161.

[15] M. Jung, C. Mertens, E. Tomat, B. Brüne, Iron as a central player and promising target in cancer progression, Int. J. Mol. Sci. 20 (2019) 273.

[16] J. Yan, L. Yang, M.F. Lin, J. Ma, X. Lu, P.S. Lee, Polydopamine spheres as active templates for convenient synthesis of various nanostructures, Small 9 (2013) 596-603.

[17] F. Ghorbani, A. Zamanian, A. Behnamghader, M.D. Joupari, A facile method to synthesize mussel-inspired polydopamine nanospheres as an active template for in situ formation of biomimetic hydroxyapatite, Mater. Sci. Eng. C 94 (2019) 729-739.

[18] X. Jiang, Y. Wang, M. Li, Selecting water-alcohol mixed solvent for synthesis of polydopamine nano-spheres using solubility parameter, Sci. Rep. 4 (2014) 1-6.

[19] A.A. Lizunova, A.A. Loshkarev, Y.M. Tokunov, V.V. Ivanov, Comparison of the results of measurements of the sizes of nanoparticles in stable colloidal solutions by the methods of acoustic spectroscopy, dynamic light scattering, and transmission electron microscopy, Meas. Tech. 59 (2017) 1151-1155.

[20] J.M. Hughes, P.M. Budd, A. Grieve, P. Dutta, K. Tiede, J. Lewis, Highly monodisperse, lanthanide-containing polystyrene nanoparticles as potential standard reference materials for environmental "nano" fate analysis, J. Appl. Polym. Sci. 132 (24) (2015) 42061. 
[21] K. Ai, Y. Liu, C. Ruan, L. Lu, G.M. Lu, Sp2 C-dominant N-doped carbon submicrometer spheres with a tunable size: a versatile platform for highly efficient oxygen-reduction catalysts, Adv Mater. 25 (2013) 998-1003.

[22] R. Batul, T. Tamanna, A. Khaliq, A. Yu, Recent progress in the biomedical applications of polydopamine nanostructures, Biomater. Sci. 5 (2017) 1204-1229.

[23] S.A. Centeno, J. Shamir, Surface enhanced Raman scattering (SERS) and FTIR characterization of the sepia melanin pigment used in works of art, J. Mol. Struct. 873 (2008) 149-159.

[24] R.A. Zangmeister, T.A. Morris, M.J. Tarlov, Characterization of polydopamine thin films deposited at short times by autoxidation of dopamine, Langmuir 29 (2013) 8619-8628.

[25] E. Nemati-Kande, H. Shekaari, S.A. Jafari, Liquid-liquid equilibrium of 1-propanol, 2-propanol, 2-methyl-2-propanol or 2-butanol + sodium sulfite + water aqueous two phase systems, Fluid Phase Equilib. 329 (2012) 42-54.
[26] M. Mohsen-Nia, H. Amiri, B. Jazi, Dielectric constants of water, methanol, ethanol, butanol and acetone: measurement and computational study, J. Solut. Chem. 39 (2010) 701-708.

[27] B. Zhao, X. Li, Y. Wang, P. Shang, Iron-dependent cell death as executioner of cancer stem cells, J. Exp. Clin. Cancer Res. 37 (2018) 79.

[28] G. Wang, G. Shen, T. Yin, In vitro assessment of deferoxamine on mesenchymal stromal cells from tumor and bone marrow, Environ. Toxicol. Pharmacol. 49 (2017) 58-64.

[29] H. Tang, D. Chen, C. Li, C. Zheng, X. Wu, Y. Zhang, W. Fei, Dual GSH-exhausting sorafenib loaded manganese-silica nanodrugs for inducing the ferroptosis of hepatocellular carcinoma cells, Int. J. Pharm. 572 (2019), 118782. 